\title{
Low Noise HTS dc-SQUID Flip-Chip Magnetometers and Gradiometers
}

\author{
M. I. Faley, U. Poppe, K. Urban, D. N. Paulson, T. N. Starr, and R. L. Fagaly
}

\begin{abstract}
We have fabricated HTS dc-SQUID flip-chip sensors with a large area multilayer flux transformers. Different layouts of the flux transformers provide a large variety of magnetometers and planar gradiometers. For the magnetometers a resolution $\sim 6 \mathrm{fT} / \sqrt{ } \mathrm{Hz}$ and the planar gradiometers a resolution of about $\sim 30 \mathrm{fT} / \mathrm{cm} \cdot \sqrt{\mathrm{Hz}}$ were routinely obtained at $77 \mathrm{~K}$. The noise was nearly white down to frequencies of few Hz. The sensors were vacuum-tight encapsulated together with a heater and a feedback coil. This makes the handling of the sensors more reproducible and convenient. Production of the magnetometers and gradiometers in small series was proven.
\end{abstract}

Index Terms-HTS, magnetometer, gradiometer, multilayer structures

\section{INTRODUCTION}

M ANY applications require measuring systems with an utmost sensitivity to magnetic fields. For example, an average $\mathrm{R}$-wave magnitude of a fetal magnetocardiogram (fMCG) signal is $\sim 2 \mathrm{pT}$. To measure the $\mathrm{fMCG}$ in a frequency bandwidth of about $100 \mathrm{~Hz}$ with a signal-to-noise ratio of $\sim 10$ one needs a system with a noise below of about $20 \mathrm{fT} / \sqrt{\mathrm{Hz}}$. Commercially available LTS dc-SQUID systems have a typical resolution $\sim 5 \mathrm{fT} / \sqrt{\mathrm{Hz}}$ (for $20 \mathrm{~mm} \times 20 \mathrm{~mm}$ pick-up loop [1] of the sensor).

A straightforward upgrade of LTS measuring systems would be the use of HTS sensors of similar resolution. This would significantly simplify the operation and drastically increase the cryogen hold time of the systems. An unexpected and astonishing result of a decade of development of HTS SQUIDs is that the field resolution of the HTS magnetometers at $77 \mathrm{~K}$ was approaching and now overlaps some LTS magnetometers operating at $4.2 \mathrm{~K}$. Cantor et al. [2] reported noise levels of $24 \mathrm{fT} / \sqrt{ } \mathrm{Hz}$ at $1 \mathrm{~Hz}$ and of $14 \mathrm{fT} / \sqrt{\mathrm{Hz}}$ at $1 \mathrm{kHz}$ using a single-layer, directly coupled magnetometer with a pickup loop $20 \mathrm{~mm} \times 20 \mathrm{~mm}$. Dantsker et al. [3] achieved a resolution of $27 \mathrm{fT} / \mathrm{J} \mathrm{Hz}$ at $1 \mathrm{~Hz}$ and $8.5 \mathrm{fT} / \sqrt{\mathrm{Hz}}$ at $1 \mathrm{kHz}$ using a flip-chip magnetometer with a dc-SQUID inductively coupled to a multilayer flux transformer having a $9 \mathrm{~mm} \times 9 \mathrm{~mm}$ pickup loop. It is obvious, that the sensitivity and reproducibility of

Manuscript received September 17, 2000.

M. I. Faley, U. Poppe, and K. Urban are with Institut für Festkörperforschung, Forschungszentrum Jülich GmbH, D-52425 Julich, Germany (telephone: +49. 2461-614366, e-mail: m. faley@fz-juelich.de).

D. N. Paulson, T. N. Starr, and R. L. Fagaly are with Tristan Technologies, Inc., San Diego, CA 92121 USA, (telephone: (858) 550-2798, e-mail: rfagaly@tristantech.com).

The corresponding author: M. I, Faley (m.faley@fz-juelich.de) the magnetometers could be additionally improved by a larger pick-up loop with the flux transformer made on a larger wafer.

To subtract high magnetic background noise one can use a gradiometric configuration of the pick-up coil. Tian et al. [4] have achieved a field gradient sensitivity of $73 \mathrm{fT} / \mathrm{cm} \sqrt{ } \mathrm{Hz}$ in the white noise region and $596 \mathrm{fT} / \mathrm{cm} \sqrt{H z}$ at $1 \mathrm{~Hz}$ with a single layer gradiometric flux antenna on a $50 \mathrm{~mm}$ Si wafer. We have demonstrated [5] a planar HTS flip-chip gradiometer having multilayer gradiometric flux antenna prepared on a $30 \mathrm{~mm}$ wafer. A resolution of $\sim 40 \mathrm{fT} / \mathrm{cm} \sqrt{\mathrm{Hz}}$ at frequencies above $10 \mathrm{~Hz}$ and $\sim 100 \mathrm{fT} / \mathrm{cm} \sqrt{\mathrm{Hz}}$ at $1 \mathrm{~Hz}$ was achieved.

In the following, we describe our present flip-chip magnetometers and gradiometers. First we outline the ramp-, bicrystal-, PMMA-, and AZ-techniques, which were used for the preparation of the dc-SQUIDs and flux transformers. We have significantly improved our early-developed [6] technology based on a nonaqueous Br-ethanol etching to achieve the high sensitivity of the sensors especially at low frequencies.

Next, we describe the layouts, capsulations, and properties of the magnetometers and gradiometers. Important is that the present stage of the technology allows a reproducible preparation of the HTS sensors with a properties at $77 \mathrm{~K}$ similar to the properties of some LTS ones operating at $4.2 \mathrm{~K}$.

\section{EXPERIMENTAL}

\section{A. Multilayer structures}

The flip-chip sensor consisted of a dc-SQUID and a multilayer flux transformer made on a separate substrate. Single- or $30^{\circ}$ bicrystal $1 \mathrm{~cm} \times 1 \mathrm{~cm} \mathrm{SrTiO}_{3}$ substrates were used for the preparation of the dc-SQUIDs. For the preparation of the flux transformers we have used single crystal $10 \mathrm{~mm} \times 10 \mathrm{~mm} \mathrm{SrTiO}_{3}$ substrates and $\varnothing 30 \mathrm{~mm}$ $\mathrm{LaAlO}_{3}$ or $\mathrm{SrTiO}_{3}$ wafers.

The structures were prepared from $\mathrm{YBa}_{2} \mathrm{Cu}_{3} \mathrm{O}_{7-\mathrm{x}}$ (YBCO) and $\mathrm{PrBa}_{2} \mathrm{Cu}_{3} \mathrm{O}_{7 \times \mathrm{x}}$ (PrBCO) $c$-oriented films by a highoxygen-pressure dc-sputtering technique [7]. The YBCO films show typical critical current densities $I_{c}$ of about $5 \times$ $10^{6} \mathrm{~A} / \mathrm{cm}^{2}$ at $77 \mathrm{~K}$ and a transition temperature $T_{c}$ above $91 \mathrm{~K}$. A high quality of ceramic films was found to be very important for good operation parameters of the HTS sensors. Films without outgrowths and with a surface roughness on 
the level of about $3 \mathrm{~nm}$ (peak-to-peak) or with a well-defined growth spirals can be prepared by this method (see Fig.1).

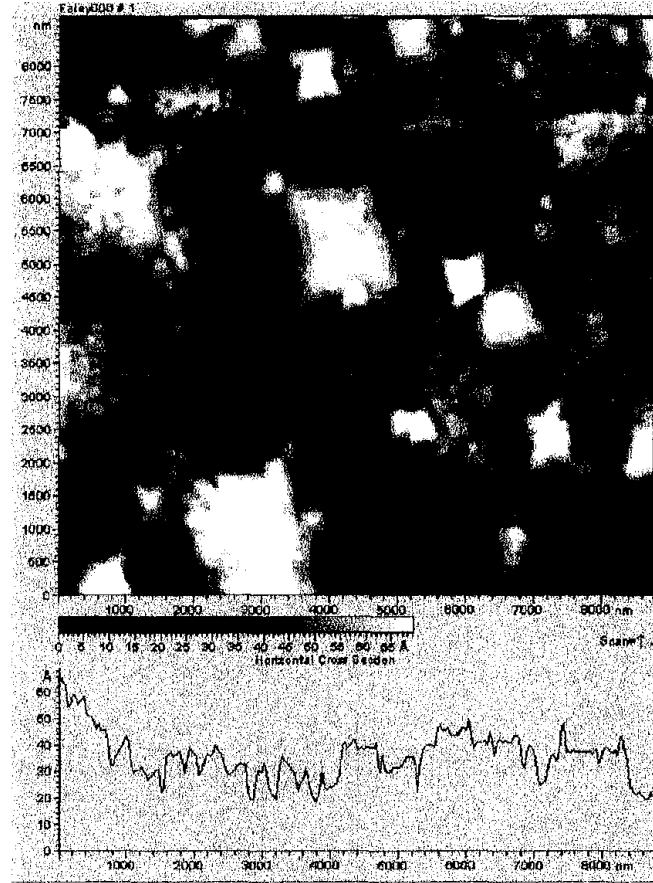

b.

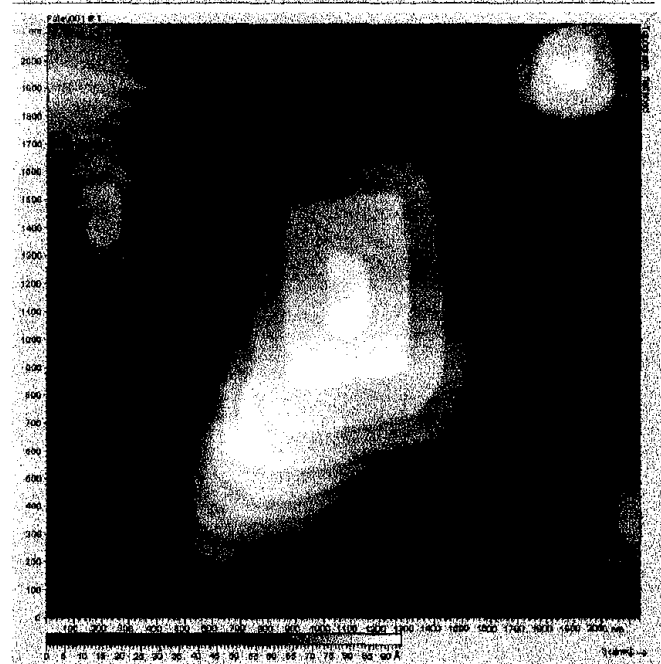

Fig. 1. AFM images of the surface of a $200 \mathrm{~nm}$ thick PrBCO film demonstrating: a) a possibility to prepare the films with $3 \mathrm{~nm}$ (peak-to-peak) surface roughness on a length of about $10 \mu \mathrm{m}$; and b) spirals, double-spirals, and islands observed on the film surface.

The HTS Josephson junctions for the dc-SQUIDs were two types: ramp-junctions [6] and $30^{\circ}$ bicrystal [8] ones. The junctions had a width of about $2 \mu \mathrm{m}$ and a critical current of about $(10-50) \mu \mathrm{A}$ at $77 \mathrm{~K}$

The multiloop flux transformers were prepared with a similar technique [6] using the PrBCO film as an insulation layer between the windings and the return strip. First two layers were patterned with deep-UV lithography using a PMMA-photoresist and a Br-ethanol etching. Top layer, containing the $\mu$-size structures was patterned with a conventional AZ-photoresist and an ion-beam etching. The PrBCO film prevents superconducting shortage and provides a normal conducting shunt of the flux transformer to avoid high frequency resonances in the coil structure.

A high quality of the crossovers and vias in the multilayer multiturn coil of the flux transformer is essential to obtain high values of the induced supercurrent. Mainly the critical current of the flux transformer limits the dynamic range of the sensors. Due to the damage-free interfaces and gently sloping edges produces by a Br-ethanol etching we have achieved a critical current for the transformer inner coil of about $100 \mathrm{~mA}$ at $77 \mathrm{~K}$. For the $8 \mathrm{~mm}$ HTM-8 magnetometer (see Fig. 2a) the pick-up loop has an inductance $\sim 20 \mathrm{nH}$. The dynamic range of the magnetometer limited by the critical current of the flux transformer is about $60 \mu \mathrm{T}$ (peakto-peak). This allows sensitive measurements with the HTM-8 magnetometer even after a movement in earth's field.
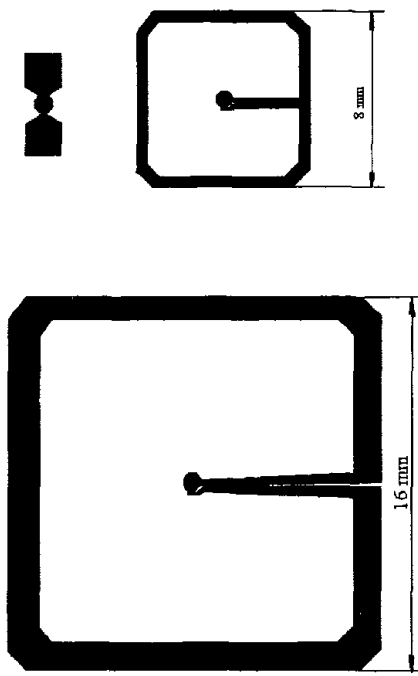

b.

Fig. 2. a) The de-SQUID and $8 \mathrm{~mm}$ multilayer flux transformer (HTM-8) used for the flip-chip magnetometers; b) The layout of the $16 \mathrm{~mm}$ flux transformer (ITM-16).

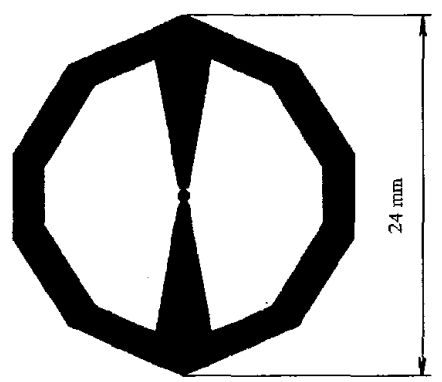

Fig. 3. The gradiometric flux transformer used for $\mathrm{HTG}-10 \mathrm{R}$. The $1 \mathrm{~mm}$ multiturn coil is placed in the middle of the flux transformers. 
Some layouts of the magnetometers and of the gradiometric flux transformers are presented in Fig. 2 and 3 respectively. The gradiometric flux transformers have a length of about $24 \mathrm{~mm}$ and a baseline of about $10 \mathrm{~mm}$.

\section{B. Capsulations}

The sensors need to be encapsulated to ensure a long and reliable service life. We enclose the sensors in fiber-glass epoxy capsulations. The capsulations are vacuum-tight sealed to prevent the HTS films from degradation by ambient atmosphere and humidity.

A modulation coil and nonmagnetic $\mathrm{Pt}$ resistor are integrated inside the capsulation. The latter one is fixed to the SQUID and serves both as a thermometer or as a heater The heater allows easy removal of trapped magnetic flux to improve the noise properties of the magnetometer. The encapsulation significantly simplifies the handling of the magnetometers during characterization, applications, and for the end-users. The capsulations can be adapted to the specific measuring systems intended for different applications. Standard dimensions of the capsulations are listed in Table 1.

Encapsulated HTM-8 and HTM-8-90 sensors were displayed in Fig. 2 of Ref. 9. The standard encapsulated gradiometer HTG-10 was displayed in Fig. 3 of Ref. 5. The capsulations for the magnetometers (HTM-8, HTM-16) and for the gradiometer (HTG-10R) are shown in Fig. 4.

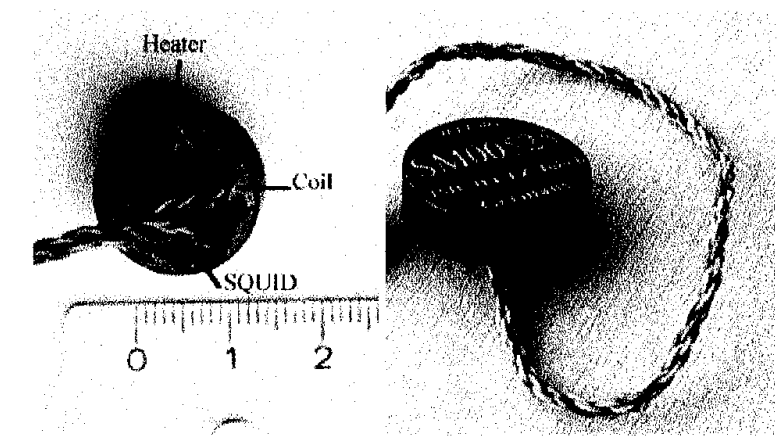

a.

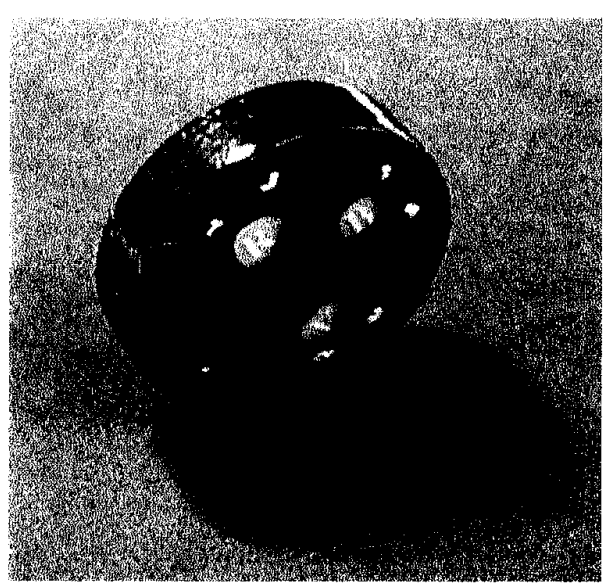

b.

Fig. 4. The standard capsulations of (a) magnetometers HTM-8 and HTM-16 and (b) gradiometer HTG-10R.
TABLE 1

SIZES OF THE STANDARD CAPSULATIONS FOR THE FLIP-CHIP SENSORS

\begin{tabular}{ccc}
\hline Sensor type & Diameter $(\mathrm{mm})$ & Thickness $(\mathrm{mm})$ \\
\hline HTM-8 & 18 & 7 \\
HTM-16 & 27 & 8 \\
HTG-10R & 40 & 10 \\
\hline
\end{tabular}

The flux noise of the sensors was measured inside an open cylinder YBCO shield in combination with a 3-layer $\mu$-metal shield.

\section{RESULTS}

We have observed that the flip-chip arrangement significantly reduces the effective SQUID inductance and provides a high effective area for the sensors. The effective inductance of the present SQUIDs inductively coupled to the flux transformers is about $100 \mathrm{pH}$. In Fig. 5 the flux sensitivity of the flip-chip magnetometers is compared with the direct-coupled magnetometers described in references [2] and [10].

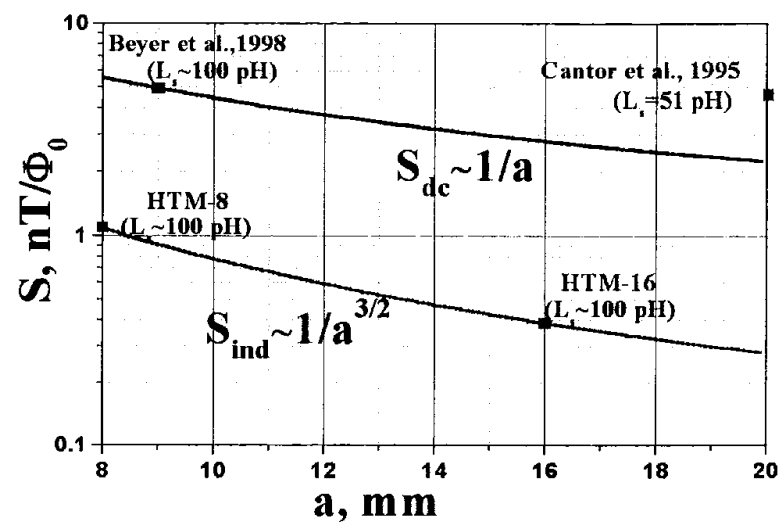

Fig. 5. Flux sensitivity "S" vs pick-up loop size "a" for direct coupled $\left(\mathbf{S}_{\mathrm{dc}}\right)$ and inductively (flip-chip) coupled ( $\left.\boldsymbol{S}_{\mathrm{ind}}\right)$ magnetometers.

The effective area $A_{\text {eff }}$ of a direct-coupled magnetometers is proportional to $A_{\text {pickup }} / L_{\text {pu }} \sim a$, where " $a$ " corresponds to size; $A_{\text {pickup }} \sim a^{2}$ to area; and $L_{\text {pickup }} \sim a$ to inductance of the pickup coil. For an inductive coupled magnetometer with an optimized multiturn input coil for the flux transformer, the effective area $\mathrm{A}_{\text {eff }} \sim \mathrm{A}_{\text {pickup }} / \mathrm{VL}_{\text {pickup }} \sim \mathrm{a}^{3 / 2}$. Flux sensitivity $\mathrm{S}\left(\mathrm{nT} / \Phi_{0}\right)=1 / \mathrm{A}_{\text {eff. }}$ In the case of a large area pickup coil the flip-chip magnetometer have a significant advantage in flux sensitivity compared to direct-coupled magnetometer.

TABLE 2

FLUX SENSITIVITIES $\Lambda$ ND NOISE ACHIEVED FOR THE FLIP-CHIP SENSORS

\begin{tabular}{ccc}
\hline \hline Sensor type & Flux sensitivity & Noise at $1 \mathrm{kHz}$ \\
\hline HTM-8 & $1 \mathrm{nT} / \Phi_{0}$ & $20 \mathrm{fT} / \mathrm{VHz}$ \\
HTM-16 & $0.4 \mathrm{nT} / \Phi_{0}$ & $6 \mathrm{fT} / \sqrt{\mathrm{Hz}}$ \\
HTG-10 & $2.2 \mathrm{nT} / \mathrm{cm}_{0}$ & $40 \mathrm{fT} / \mathrm{cm} / \mathrm{Hz}$ \\
HTG-10R & $1.7 \mathrm{nT} / \mathrm{cm}_{0}$ & $30 \mathrm{fT} / \mathrm{cm} / \mathrm{Hz}$ \\
\hline \hline
\end{tabular}




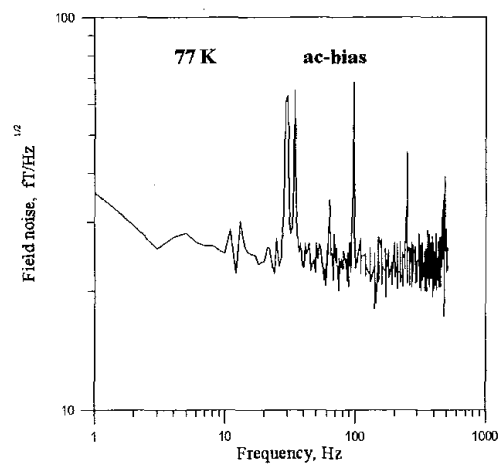

Fig. 6. Noise spectrum for HTM-8, measured inside a 3-layer $\mu$-metal shield and a superconducting shield.

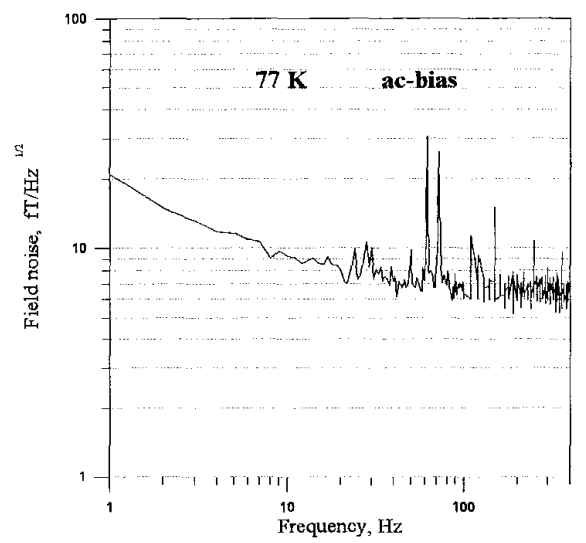

Fig. 7. Noise spectrum for HTM-16, measured inside a 3-layer $\mu$-matal shield and a superconducting shield.

Typical sensitivities and noise achieved for the flip-chip sensors are listed in the Table 2. The noise spectra for the HTM-8 and HTM-16 magnetometers are presented in Fig. 6 and 7 , correspondingly. The noise spectrum for HTG-10 gradiometer was demonstrated in Ref. 5. Recently, the noise data of our magnetometers were independently confirmed using STL-electronics [11] in the new shielded room of PTB (Berlin).

The flux noise of the HTM-16 is about $15 \mu \Phi_{0} / \sqrt{ } \mathrm{Hz}$. For the $100 \mathrm{pH} 30^{\circ}$ bicrystal HTS dc-SQUIDs a flux noise $\sim 5$ $\mu \Phi_{0} / \sqrt{ } \mathrm{Hz}$ was achieved (see, e.g., [8]). This means that there is a significant potential for furthers improvements in field and field gradient resolution for our sensors even with the present layouts. A resolution of the HTM-16 magnetometers of about $2 \mathrm{fT} / \sqrt{\mathrm{Hz}}$ seems possible in future. Similar field noise originates from $\mathrm{Al}$-coated Mylar foil used for a thermal insulation in fiber-glass cryostats intended for operation with liquid helium $(4.2 \mathrm{~K})$. Improvement of the liquid nitrogen cryostats can be achieved using a modified thermal insulation without such foil. Thus, one can expect to achieve a field resolution below $1 \mathrm{fT} / \sqrt{\mathrm{Hz}}$ for the HTS system operating at $77 \mathrm{~K}$. For this purpose magnetometers with a larger pickup coils $(\sim 30 \mathrm{~mm})$ need to be developed.

\section{SUMMARY}

The HTS sensors reported here have a sensitivity and reliability sufficient for many applications. The magnetometers achieved a white noise of about $6 \mathrm{fT} / \sqrt{\mathrm{Hz}}$ and the planar gradiometers achieved a gradient resolution of about $30 \mathrm{fT} / \mathrm{cm} \cdot \sqrt{\mathrm{Hz}}$ at $77 \mathrm{~K}$. The resolution of the sensors can be further improved in the future. The magnetometers and gradiometers are able to operate without magnetic shielding. The user-friendly capsulation for the sensors makes handling of the sensors safe, reproducible, and convenient. Production of both magnetometers and gradiometers in small numbers has been demonstrated and is sufficient for the construction of multichannel HTS systems.

\section{ACKNOWLEDGMENT}

The authors gratefully acknowledge $\mathrm{R}$. Wagner for official organization and support of our co-operation and the technical assistance of W. Evers.

\section{REFERENCES}

[1] V. Diekmann, R. Jürgens, W. Becker, H. Elias, W. Ludwig, and W. Vodel, "RF-SQUID to DC-SQUID upgrade of a 28-channel magnetoencephalography (MEG) system," Meas. Sci. Technol. vol. 7, pp. 844 852, 1996.

[2] R. Cantor, L. P. Lee, M. Teepe, V. Vinetskiy, and J. Longo, "Low-noise single-layer $\mathrm{YBa}_{2} \mathrm{Cu}_{3} \mathrm{O}_{7}$ DC-SQUID magnetometers at $77 \mathrm{~K}$," IEEE Transactions on Appl.Supercond. vol.5, No. 2, p. 2927, 1995.

[3] E. Dantsker, F. Ludwig, R. Kleiner, J. Clarke, M. Teepe, L. P. Lee, N. $\mathrm{McN}$. Alford, and $\mathrm{T}$. Button, "Addendum: "Low noise $\mathrm{YBa}_{2} \mathrm{Cu}_{3} \mathrm{O}_{7 \times \mathrm{x}}-$ $\mathrm{SrTiO}_{3}-\mathrm{YBa}_{2} \mathrm{Cu}_{3} \mathrm{O}_{7 \cdot \mathrm{x}}$ multilayers for improved superconducting magnetometers","'Appl. Phys. Lett., vol.67, No. 5, pp. 725-726, 1995.

[4] Y. J. Tian, S. Linzen, F. Schmidl, L. Dörrer, R. Weidl, and P. Seidel, "High-Tc directly coupled direct current SQUID gradiometer with flipdhip flux transformer," Appl. Phys. Lett., vol. 74, No. 9, pp. 1302-1304, 1999.

[5] M. I. Faley, H. Soltner, U. Poppe, K. Urban, D. N. Paulson, T. N. Starr, and R. L. Fagaly, "HTS de-SQUID with gradiometric multilayer flux transformer," Inst. Phys. Conf. Ser., No.167, vol. 2, p.509-512, 2000. Paper presented at Applied Superconductivity, Spain, 14-17 September 1999.

[6] M. I. Faley, U. Poppe, H. Soltner, C. L. Jia, M. Siegel, and K. Urban, "Josephson junctions, interconnects and crossovers on chemically etched edges of $\mathrm{YBa}_{2} \mathrm{Cu}_{3} \mathrm{O}_{7-\mathrm{x}}$," Appl. Phys. Lett., vol. 63, No. 15, pp. 2138$2140,1993$.

[7] U. Poppe, N. Klein, U. Dähne, H. Soltner, C. L. Jia, B. Kabius, K. Urban, A. Lubig, K. Schmid, S. Hensen, S. Orbach, G. Müller, and H. Piel, "Low-resistivity epitaxial $\mathrm{YBa}_{2} \mathrm{Cu}_{3} \mathrm{O}_{7-\mathrm{x}}$ thin films with improved microstructure and reduced microwave losses," J. Appl. Phys., vol. 71, p. $5572,1992$.

[8] K. Enpuku, T. Minotani, F. Shiraishi, A. Kandori, and S. Kawakami, "High $\mathrm{T}_{\mathrm{c}}$ de SQUID Utilizing Bicrystal Junctions with 30 Degree Misorientation Angle," IEEE Transactions on Appl.Supercond., vol. 9, No. 2, pp. 3109-3112, 1999.

[9] M. I. Faley, U. Poppe, K. Urban, E. Zimmermann, W. Glaas, H. Halling, M. Bick, D. N. Paulson, T. N. Starr, and R. L. Fagaly, "Operation of the HTS dc-SQUID Sensors in High Magnetic Fields," IEEE Transactions on Appl.Supercond., vol. 9, No. 2, pp. 3386-3391 1999.

[10] J. Beyer, D. Drung, F. Ludwig, T. Minotani, and K. Enpuku, "Low-noise $\mathrm{YBa}_{2} \mathrm{Cu}_{3} \mathrm{O}_{7}$ single layer de superconducting quantum interference device (SQUID) magnetometer based on bicrystal junctions with $30^{\circ}$ misorientation angle," Appl. Phys. Lett., vol. 72, No. 2, pp. 203-205, 1998.

[11] STL Systemtechnik Ludwig GmbH, Konstanz, Germany. 